\title{
Extended coupled-cluster treatment of correlations in quantum magnets
}

\author{
J. Rosenfeld, N. E. Ligterink, and R. F. Bishop* \\ Department of Physics, University of Manchester, Institute of Science and Technology (UMIST), P.O. Box 88, \\ Manchester, M60 1QD, United Kingdom
}

(Received 20 January 1999)

\begin{abstract}
The spin-half $X X Z$ model on the linear chain and the square lattice are examined with the extended coupled-cluster method (ECCM) of quantum many-body theory. We are able to describe both the IsingHeisenberg phase and the $X Y$-Heisenberg phase, starting from known wave functions in the Ising limit and at the phase transition point between the $X Y$-Heisenberg and ferromagnetic phases, respectively, and by systematically incorporating correlations on top of them. The ECCM yields good numerical results via a diagrammatic approach, which makes the numerical implementation of higher-order truncation schemes feasible. In particular, the best nonextrapolated coupled-cluster result for the sublattice magnetization is obtained, which indicates the employment of an improved wave function. Furthermore, the ECCM finds the expected qualitatively different behaviors of the linear-chain and square-lattice cases. [S0163-1829(99)02530-8]
\end{abstract}

\section{INTRODUCTION}

The extended coupled-cluster method ${ }^{1-5}$ (ECCM) has not previously been applied to lattice spin systems, unlike the normal coupled-cluster method ${ }^{3-13}$ (NCCM), which is a restricted version of the ECCM at a given level of approximation, and which has been widely implemented for these systems. The primary aim of this paper is to apply the ECCM to the spin-half anisotropic Heisenberg (or $X X Z$ ) model, in order to obtain numerical results for the ground-state energy and the sublattice magnetization, and thereby to investigate the usefulness of the method in the study of quantum phase transitions. The ECCM and NCCM are $a b$ initio techniques of microscopic quantum many-body theory, generically known as the coupled-cluster method (CCM). The ECCM, in contrast to the NCCM, completely characterizes a system in terms of a set of basic amplitudes, all of which are linkedcluster quantities.

The two-dimensional spin-half $X X Z$ model is expected to have a second-order phase transition at the isotropic point $\Delta=1$, where the system is in a unique critical phase. The transition is expected to be accompanied by some change of symmetry of the ground-state wave function. In particular, Laughlin $^{14}$ speculates that the physics of the isotropic point can be understood as a gauge theory with massless excitations. In general, theoretical study of the isotropic point requires some prior knowledge of the phases on either side of the isotropic point. In practice, the ordering and symmetry of the known wave functions in these phases influence the predicted state at the isotropic point. Therefore, the second aim of this paper is to examine how the CCM, in particular, is affected by this universal problem in the study of phase transitions.

The general $X X Z$ model Hamiltonian has $\mathrm{Z}(2) \otimes \mathrm{U}(1)_{x y}$ symmetry, except at the isotropic point where it has $\mathrm{SU}(2)$ symmetry. For the square-lattice case the ground-state wave function is expected to yield broken $\mathrm{U}(1)_{x y}$ symmetry in the $X Y$-like region, and there is broken $\mathrm{Z}(2)$ symmetry in the Ising limit. Approaching the isotropic point from either side with the CCM yields different ground-state wave functions at the isotropic point itself, which is due to the special nature of the isotropic point.

Any CCM calculation on spin systems involves adding correlations between spins, on top of those already contained in a separately chosen model or reference state, in order to produce the true quantum-mechanical ground-state wave function. Therefore, the important physical characteristics of a system are incorporated into the CCM by the choice of the model state and by the inclusion of particular configurations for the correlations. Hence, we can now refine and reformulate our second aim to be an examination of the effect of the choice of model state. In particular, we shall be interested in the interplay between the symmetry of the model state and the symmetry of the Hamiltonian influencing the groundstate wave function. This is exemplified by the CCM treatment of the linear-chain case, which yields artificially broken $\mathrm{U}(1)_{x y}$ and $\mathrm{SU}(2)$ symmetries. This can only be due to the choice of model state, since exact results show that the symmetry of the $X X Z$ model Hamiltonian is not broken in the $X Y$-like region and at the isotropic point.

The primary aim of this paper is motivated by numerical evidence, from a number of techniques, of long-range order (LRO) at the isotropic point for the square-lattice case. $\mathrm{Nu}-$ merical results from a wide variety of techniques such as spin-wave theory, ${ }^{15-17}$ high-accuracy quantum Monte Carlo (QMC) simulations, ${ }^{18}$ and series expansions, ${ }^{19,20}$ yield a sublattice magnetization at the isotropic point of approximately $61-62 \%$ of the classical value arising from perfect Néel ordering. A priori, the ECCM is expected to perform better than the NCCM for systems that undergo global changes. This is put to the test, where we pay special attention to the possible underestimation of quantum fluctuations due to the choice of ordered state from which the calculation starts, ${ }^{21}$ by using several different such ordered states.

Rigorous results from the Bethe ansatz $\mathrm{z}^{22-24}$ for the spinhalf anisotropic Heisenberg antiferromagnet on the linear chain provide a measure of the effectiveness of the ECCM. However, we know in advance that the nature of the transition in the linear chain is very subtle, and quantum fluctuations present in this case are known to destroy Néel LRO 
completely at the isotropic point. ${ }^{25}$ Therefore, a priori, one would expect the ECCM to be more effective for the squarelattice case, where the ground state will be closer to the classical uncorrelated state.

Numerical results for the ground states of models in one and two dimensions are expected to be qualitatively different. ${ }^{26}$ By contrast with the NCCM, which has previously been applied, for example, to the spin-half $X X Z$ model $^{27}$ and the nonlinear $\sigma$ model, ${ }^{28}$ the ECCM is expected to yield numerical evidence of this difference. As similar calculations are performed for any lattice dimensionality in the CCM, the qualitatively different behavior of the solutions that we report in this paper for the two cases using the ECCM is not simply an artifact of the technique.

In a diagrammatic implementation of both CCM techniques, the ECCM, at any level of truncation, produces diagrams in greater abundance and of greater complexity than the NCCM, as shown in Sec. IV. Unlike in its NCCM counterpart, it is possible within the ECCM formalism to define a spin-spin correlation function which is fully consistent with the corresponding definition of the magnetic order parameter, at all orders of truncation. Such properties of the ECCM make it attractive for describing correlation effects in physical systems, and particularly to study their quantum phase transitions and quantum order.

The CCM has been used very successfully ${ }^{6-13}$ to calculate the zero-temperature properties of a wide variety of extended many-body systems, including, for example, atoms and molecules, nuclear matter and finite nuclei, the electron gas, and lattice gauge field theory, as well as spin-lattice systems..$^{27,29-33}$. In this paper, we present evidence from numerical results that demonstrates the particular superiority of the ECCM over the NCCM, in practice, to study such global properties of spin systems as their quantum (zerotemperature) phase transitions.

\section{EXTENDED COUPLED CLUSTER METHOD FORMALISM}

Since detailed descriptions of the CCM formulation have been given elsewhere, ${ }^{1-13}$ only the essential components will be given here. Hubbard ${ }^{34}$ was one of the first to emphasize the importance of an exponential parametrization of the exact ground-state ket wave function $|\psi\rangle$ of an interacting many-body system,

$$
|\psi\rangle=e^{S}|\phi\rangle, \quad S=\sum_{I}^{\prime} s_{I} C_{I}^{\dagger},
$$

in terms of a model state $|\phi\rangle$ which is not orthogonal to the exact ground-state ket wave function $|\psi\rangle$. The correlation operator $S$ in Eq. (2.1) is decomposed solely in terms of a complete set of mutually commuting, linked, multiconfigurational creation operators $C_{I}^{\dagger}$ defined in terms of a complete set of many-body configurations $\{I\}$. These are, in turn, defined by a set index $I$, which, for the spin-lattice systems under consideration, describes the set of spins which are flipped with respect to those contained in the suitably chosen, normalized, model state $|\phi\rangle$. The prime on the sum in Eq. (2.1) excludes the null set, $I \rightarrow 0$, corresponding to the identity operator, $C_{0}^{\dagger} \equiv 1$. We note that $C_{I}|\phi\rangle=0, \forall I \neq 0$, and hence the state $|\psi\rangle$ obeys the intermediate normalization condition $\langle\phi \mid \psi\rangle=1$.

Arponen ${ }^{1}$ has shown how two distinct CCM parametrizations of the exact ground bra state $\langle\widetilde{\psi}|$ can be given, which yield the NCCM and the ECCM, respectively. The NCCM parametrization of the ground bra state is given as

$$
\langle\widetilde{\psi}|=\langle\phi| \widetilde{S} e^{-S}, \quad \widetilde{S}=1+\sum_{I}{ }^{\prime} \tilde{S}_{I} C_{I},
$$

whereas the corresponding ECCM parametrization is given as

$$
\langle\widetilde{\psi}|=\langle\phi| e^{S^{\prime \prime}} e^{-S}, \quad S^{\prime \prime}=\sum_{I}^{\prime} s_{I}^{\prime \prime} C_{I} .
$$

Both $\widetilde{S}$ and $S^{\prime \prime}$ are constructed wholly in terms of multiconfigurational destruction operators, defined with respect to the model state $|\phi\rangle$. These are simply the Hermitian adjoints $C_{I}$ of the corresponding creation operators $C_{I}^{\dagger}$ in Eq. (2.1). Furthermore, both parametrizations satisfy the normalization condition $\langle\widetilde{\psi} \mid \psi\rangle=1$. Although $\langle\widetilde{\psi}|=\langle\psi| /\langle\psi \mid \psi\rangle$ formally, this relation may not be preserved when truncations are made, as explained below, in either parametrization.

The energy functional $\bar{H}_{E}$ in the ECCM formulation is thus given by

$$
\bar{H}_{E} \equiv\left\langle\phi\left|e^{S^{\prime \prime}} e^{-S} H e^{S}\right| \phi\right\rangle .
$$

The similarity transformed Hamiltonian $e^{-S} H e^{S}$ may be expressed as the usual nested commutator expansion,

$$
e^{-S} H e^{S}=H+[H, S]+\frac{1}{2 !}[[H, S], S]+\cdots .
$$

Since $S$ is composed wholly of mutually commuting creation operators, the similarity transform in Eq. (2.5) only retains terms in which all amplitudes $s_{I}$ are linked to the Hamiltonian. Furthermore, provided that the Hamiltonian is finite order in the single-body operators, the expansion always terminates at finite order. Therefore, once the correlation operator $S$ is approximated, no further truncations are necessary.

Formally, the bra-state parametrizations in both the ECCM and NCCM formulations still preserve Hermiticity. Explicitly, we have the relations

$$
\langle\phi| \widetilde{S}=\langle\phi| e^{S^{\prime \prime}}=\frac{\langle\phi| e^{S^{\dagger}} e^{S}}{\left\langle\phi\left|e^{S^{\dagger}} e^{S}\right| \phi\right\rangle},
$$

which are a consequence of satisfying the normalization condition $\langle\widetilde{\psi} \mid \psi\rangle=1$. Although the manifest Hermiticity is usually sacrificed at any level of truncation, the distinct bra-state parametrizations produce fully linked expectation values in Eq. (2.4), as they incorporate the similarity transform, and both $S^{\prime \prime}$ and $\widetilde{S}$ are composed wholly of destruction operators.

The double-exponential structure of the ECCM formalism implies that Eq. (2.4) can be expressed in terms of the amplitudes $\left\{s_{I}, s_{I}^{\prime \prime}\right\}$ via a double-similarity transform, and that both of these sets of ECCM amplitudes are linked-cluster quantities. However, the NCCM formalism only allows a 
single-exponential structure in terms of the amplitudes $\left\{s_{I}\right\}$. Although the amplitudes $\left\{s_{I}\right\}$ are linked-cluster quantities, the amplitudes $\left\{\tilde{s}_{I}\right\}$ are not.

An arbitrary expectation value $\bar{A}$ can thus be expressed in the ECCM and the NCCM, respectively, as

$$
\begin{gathered}
\bar{A}_{E} \equiv\left\langle\phi\left|e^{S^{\prime \prime}} e^{-S} A e^{S}\right| \phi\right\rangle=\bar{A}_{E}\left(\left\{s_{I}\right\},\left\{s_{I}^{\prime \prime}\right\}\right), \\
\bar{A}_{N} \equiv\left\langle\phi\left|\widetilde{S} e^{-S} A e^{S}\right| \phi\right\rangle=\bar{A}_{N}\left(\left\{s_{I}\right\},\left\{\tilde{s}_{I}\right\}\right) .
\end{gathered}
$$

In practice, either version of the CCM can only be implemented by truncating the expansions in Eqs. (2.1)-(2.3) by retaining only a finite or infinite subset of the complete set of configurations indices $\{I\}$. In this paper the SUB2- $n$ approximation scheme is employed, which retains all configurations with up to two-body correlations between spins which are no more than a certain distance apart, specified by the index $n$. The details of the approximation scheme are given explicitly in Sec. IV where the diagrammatic approach is described.

All ground-state properties can be determined in the ECCM and the NCCM, respectively, by the stationary principle

$$
\frac{\partial \bar{H}_{E}}{\partial s_{I}}=0=\frac{\partial \bar{H}_{E}}{\partial s_{I}^{\prime \prime}}, \quad \frac{\partial \bar{H}_{N}}{\partial s_{I}}=0=\frac{\partial \bar{H}_{N}}{\partial \tilde{s}_{I}},
$$

in the case where $|\psi\rangle$ and $\langle\widetilde{\psi}|$ correspond to the ground state. We remark, however, that due to the lack of manifest Hermiticity between $\langle\widetilde{\psi}|$ and $|\psi\rangle$ at a given level of truncation, the resulting stationary values of $\bar{H}_{E}$ and $\bar{H}_{N}$ are not necessarily upper bounds to the ground-state energy.

We note that the NCCM equations at a given order can formally be extracted from the corresponding ECCM equations at the same order by truncating the expansion of the exponentiated correlation operator $e^{S^{\prime \prime}}$ at first order and performing the substitution $s_{I}^{\prime \prime} \rightarrow \tilde{s}_{I}$. In practice, the resulting ECCM equations from a particular truncation scheme are highly nonlinear and, therefore, of greater complexity than their NCCM counterparts. However, the diagrammatic representation of the formalism which we present here makes tractable the numerical implementation of high-order approximation schemes.

Very importantly, observables in the ECCM, which quantify the global behavior of a system in terms of its long-range order obey the cluster property. Thus, for example, we have the very general relation

$$
\lim _{\left|\mathbf{r}-\mathbf{r}^{\prime}\right| \rightarrow \infty} \overline{A_{\mathbf{r}} B_{\mathbf{r}^{\prime}}}=\bar{A}_{\mathbf{r}} \bar{B}_{\mathbf{r}^{\prime}}
$$

where $A_{\mathbf{r}}$ and $B_{\mathbf{r}^{\prime}}$ are single-body operators acting at defined sites $\mathbf{r}$ and $\mathbf{r}^{\prime}$, respectively. This condition is preserved by the ECCM due to the exact multiplicative separability of both the bra-state and ket-state parametrizations in the corresponding large-distance limit; however, the index set $\{I\}$ is truncated. Consequently, the long-range order of physical systems can be examined unambiguously via the ECCM parametrization of their correlation functions. As the NCCM bra-state parametrization is not multiplicatively separable, however, the physical condition in Eq. (2.10) will not in general hold at arbitrary levels of truncation within this scheme.

\section{TRANSFORMATION OF THE SPIN-HALF $X X Z$ MODEL HAMILTONIAN FOR THE LINEAR CHAIN AND SQUARE LATTICE}

\section{A. CCM approach to the spin-half $X X Z$ model Hamiltonian}

The spin-half $X X Z$ model Hamiltonian is given by

$$
H=+\frac{1}{4} \sum_{i, \rho}\left[\Delta \sigma_{i}^{z} \sigma_{i+\rho}^{z}+\sigma_{i}^{x} \sigma_{i+\rho}^{x}+\sigma_{i}^{y} \sigma_{i+\rho}^{y}\right],
$$

where $\sigma_{i}^{\alpha}, \alpha=x, y, z$, are the Pauli spin matrices, $\Delta$ is the anisotropy parameter, and the summation is over all the $N$ lattice sites denoted by $i$ and over each of the nearestneighbor vectors denoted by $\rho$. We note that the linear chain and the square lattice are both bipartite lattices which can be split into two identical sublattices, which we denote as the $A$ and $B$ sublattices. Thus, each nearest-neighbor site to a site on the $A$ sublattice is on the $B$ sublattice, and vice versa.

In the Ising limit $\Delta \rightarrow \infty$, the $\Delta$-dependent term in the Hamiltonian of Eq. (3.1) becomes dominant and the classical $z$-aligned Néel state is the eigenstate that yields the lowest energy. The $z$-aligned Néel state has nearest-neighbor spins ordered antiparallel to one another in the $z$ direction, as illustrated below:

$$
|\phi\rangle=\bigotimes_{k \in A}|\uparrow\rangle_{k} \bigotimes_{l \in B}|\downarrow\rangle_{l}
$$

in a notation in which the $z$ axis points vertically upwards. However, for all finite values of $\Delta$ the terms in the $x$ and $y$ directions in the Hamiltonian in Eq. (3.1) come into play, and the $z$-aligned Néel state is no longer an eigenstate of the Hamiltonian. The ground state for even $N$ now consists of a particular linear combination of all possible configurations with N/2 up-pointing spins and N/2 down-pointing spins. All the configurations apart from the classical state which are present in the exact state are considered to be quantum fluctuations upon that state. At the isotropic point the Hamiltonian in Eq. (3.1) becomes rotationally invariant, such that the expectation value of an arbitrary spin is the same for any direction.

For $-1<\Delta<1$ the true classical ground state can be any one of an infinite number of degenerate Néel states with nearest-neighbor spins restricted to align antiparallel to one another in any direction in the $X Y$ plane. Schematically, we write

$$
|\phi\rangle=\bigotimes_{k \in A}|\leftarrow\rangle_{k} \bigotimes_{l \in B}|\rightarrow\rangle_{l},
$$

choosing, say, the $x$ direction to be the alignment axis, and in a notation in which the positive $x$ axis points horizontally to the right. We note that there exists a trivial transformation of the Hamiltonian in Eq. (3.1) at the phase transition point $\Delta$ $=-1$ between the $X Y$-Heisenberg and ferromagnetic phases (henceforth referred to as the ferromagnetic point), with the $x$-aligned Néel state as eigenstate, to the ferromagnetic Heisenberg Hamiltonian, with the $x$-aligned ferromagnetic state as eigenstate. Therefore, at $\Delta=-1$ the $x$-aligned Néel state is the true ground state and this point is chosen to be the 
initial point of the CCM calculation in the entire regime $-1<\Delta<1$. One imagines that the $x$-aligned Néel model state is close to the true ground state in the neighborhood of this point.

\section{B. Transformation of the Hamiltonian in terms of a canted model state}

In order to produce a ground-state solution that can describe both the $X Y$-like $(-1<\Delta<1)$ and Ising-like $(\Delta$ $>1$ ) regions of the $X X Z$ model, a canted model state is introduced. The canted model state $\left|\phi_{c}\right\rangle \equiv\left|\phi\left(\xi_{A}, \xi_{B}\right)\right\rangle$ consists of spins on the $A$ sublattice pointing in one particular direction and spins on the $B$ sublattice also pointing in another particular direction, defined by the spin-half spin vectors $\xi_{A}$ and $\xi_{B}$, respectively.

In order to calculate the expectation values of arbitrary observables in the ECCM, an arbitrary rotation of the local spin axes is performed about the $y$ axis on each sublattice, resulting in a notional rotation of the spins in the canted model state to the down position in the direction of the negative $z$ axis,

$$
U\left|\phi\left(\xi_{A}, \xi_{B}\right)\right\rangle=\bigotimes_{i=1}^{N}|\downarrow\rangle_{i} \equiv|F\rangle,
$$

where $U$ is a product of unitary matrices, which causes the spins in the canted model $\left|\phi_{c}\right\rangle$ to undergo a passive rotation such that they all point downwards in the rotated local frames. Hence, the ECCM expectation value for an observable $A$ in Eq. (2.7) can be expressed with respect to the unrotated canted model state in the form

$$
\bar{A}=\left\langle\phi_{c}\left|U^{\dagger} e^{S^{\prime \prime}} e^{-S}\left(U A U^{\dagger}\right) e^{S} U\right| \phi_{c}\right\rangle .
$$

The rotation matrices for the canted model state on the $A$ sublattice and $B$ sublattice are given by

$$
U_{J} \equiv \exp \left(-i \theta_{J} \frac{\sigma^{y}}{2}\right)=\cos \left(\frac{\theta_{J}}{2}\right) 1-i \sin \left(\frac{\theta_{J}}{2}\right) \sigma^{y}, \quad J=A, B,
$$

where each rotation is chosen to be performed about the $y$ axis in the $X Z$ plane. This involves no loss of generality, since all directions in the $X Y$ plane are equivalent, due to the $\mathrm{U}(1)_{x y}$ symmetry of the Hamiltonian in Eq. (3.1).

Now, the transformed Hamiltonian arising from Eq. (3.1) can be written as

$$
\begin{gathered}
H^{T} \equiv U H U^{\dagger}=+\frac{1}{2} \sum_{\langle k, l\rangle}\left[\Delta\left(U_{A} \sigma_{k}^{z} U_{A}^{\dagger}\right)\left(U_{B} \sigma_{l}^{z} U_{B}^{\dagger}\right)\right. \\
\left.+\left(U_{A} \sigma_{k}^{x} U_{A}^{\dagger}\right)\left(U_{B} \sigma_{l}^{x} U_{B}^{\dagger}\right)+\left(U_{A} \sigma_{k}^{y} U_{A}^{\dagger}\right)\left(U_{B} \sigma_{l}^{y} U_{B}^{\dagger}\right)\right], \\
k \in A, \quad l \in B,
\end{gathered}
$$

where $k$ and $l$ are nearest-neighbor vectors.

There are two degrees of freedom, $\theta_{A}$ and $\theta_{B}$, present in the transformed Hamiltonian $H^{T}$, which are conveniently expressed via an equivalent set of relative and total orientation parameters

$$
\alpha \equiv\left(\theta_{B}-\theta_{A}\right), \quad \beta \equiv-\left(\theta_{A}+\theta_{B}\right) .
$$

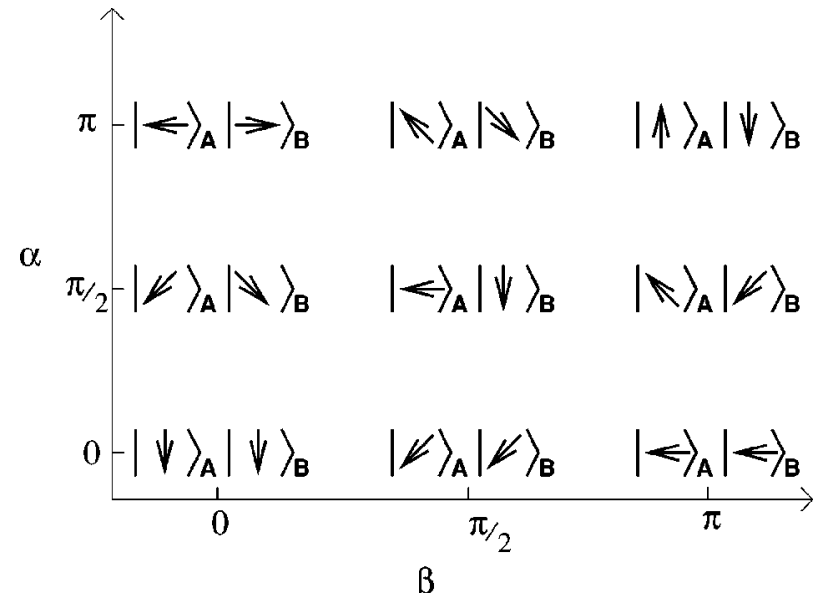

FIG. 1. Various model states canted in the $X Z$ plane, defined by the angles $\alpha$ and $\beta$, are shown in the original global coordinate frame, in a notation in which the positive $x$ axis points to the right and the positive $z$ axis points upwards. Note that the states with $\alpha$ $=\pi$ are all Néel states and those with $\alpha=0$ are all ferromagnetic states.

Particular values of $\alpha$ and $\beta$ lead to specific canted model states, as shown in Fig. 1. The single-spin creation and destruction operators are defined as $\sigma^{ \pm} \equiv \frac{1}{2}\left(\sigma^{x} \pm i \sigma^{y}\right)$ for all sites on the lattice, once the spins have all been rotated into the down position, as given in Eq. (3.4). Thus, $H^{T}$ in Eq. (3.7) can be expressed in the form

$$
H^{T}=+\frac{1}{8} \sum_{i, \rho} \sum_{p, q} T_{p q} \sigma_{i}^{p} \sigma_{i+\rho}^{q},
$$

where $p, q \in\{z,+,-\}$ and $T_{p q}$ are functions of $\alpha, \beta$, and $\Delta$. Due to the Hermiticity of the Hamiltonian, the factors $T_{p q}$ in Eq. (3.9) satisfy the relation $T_{+z}=T_{-z}^{*}$. Moreover, the spatial symmetry with respect to which lattice sites the spin operators act upon yields the relations:

$$
T_{z+}=T_{+z}, \quad T_{z-}=T_{-z}, \quad T_{+-}=T_{-+} .
$$

Finally, the transformed Hamiltonian with a canted model state can be expressed as

$$
\begin{aligned}
H^{T}= & +\frac{1}{8} \sum_{i, \rho}\left\{\frac { 1 } { 2 } [ ( 1 + \Delta ) \operatorname { c o s } \alpha + ( 1 - \Delta ) \operatorname { c o s } \beta - 2 ] \left(\sigma_{i}^{+} \sigma_{i+\rho}^{+}\right.\right. \\
& \left.+\sigma_{i}^{-} \sigma_{i+\rho}^{-}\right)+\frac{1}{2}[(1+\Delta) \cos \alpha+(\Delta-1) \cos \beta] \sigma_{i}^{z} \sigma_{i+\rho}^{z} \\
& +[(1+\Delta) \cos \alpha+(1-\Delta) \cos \beta+2] \sigma_{i}^{+} \sigma_{i+\rho}^{-} \\
& \left.+(1-\Delta) \sin \beta\left(\sigma_{i}^{z} \sigma_{i+\rho}^{+}+\sigma_{i}^{z} \sigma_{i+\rho}^{-}\right)\right\} .
\end{aligned}
$$

\section{Mean-field calculation}

A mean-field calculation provides a guideline for the model-state analysis in the correlated CCM calculation, which will be performed in Sec. VI. We restrict our meanfield calculation here to finding an optimal state of the form of the canted model state $\left|\phi\left(\xi_{A}, \xi_{B}\right)\right\rangle$, in which no other correlations are present apart from those between the two 


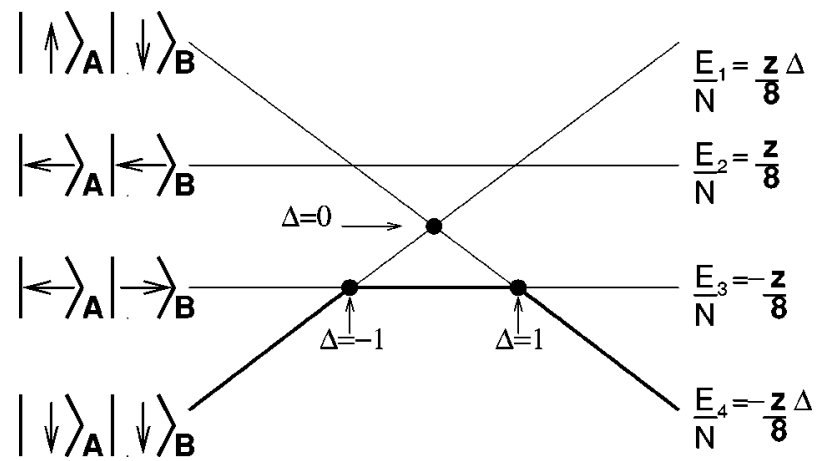

FIG. 2. The stationary energy eigenvalues $E / N$ as a function of the anisotropy $\Delta$ for the mean-field case. The ground-state energy is denoted by a thick line.

sublattices implied by the angles $\alpha$ and $\beta$. Consequently, the mean-field energy $E$, which is given by

$$
E=\left\langle\phi_{c}|H| \phi_{c}\right\rangle=\left\langle F\left|H^{T}\right| F\right\rangle,
$$

yields the following expression:

$$
\frac{E}{N}=\frac{z}{16}[(\Delta+1) \cos \alpha+(\Delta-1) \cos \beta],
$$

where $N$ is the number of lattice sites and $z$ is the coordination number of the lattice. The factor $T_{z z}$ in the Hamiltonian in Eq. (3.9) yields the only nonzero, diagonal contribution from Eq. (3.12). The stationary values of $T_{z z}$ with respect to the angles $\alpha$ and $\beta$ yield the solutions for the energy $E$, which are shown in Fig. 2. Figure 2 also shows that the model states which make up the ground state in the meanfield case are the $z$-aligned Néel state for $\Delta \geqslant 1$, the $x$-aligned (or $y$-aligned) Néel state for $-1 \leqslant \Delta \leqslant 1$, and the $z$-aligned ferromagnetic state for $\Delta<-1$.

\section{DIAGRAMMATIC APPROACH}

\section{A. Diagrammatic technique}

The terms arising from a CCM calculation have been represented diagrammatically in previous work. ${ }^{29,35}$ For example, Roger and Hetherington ${ }^{29}$ represent the terms arising from the NCCM energy functional diagrammatically simply for convenience. Unlike our representation, shown in Fig. 3, their diagrams do not include the destruction operators from the bra-state parametrization. Hence, diagrams are retained in Ref. 29, which do not contribute to the energy functional,

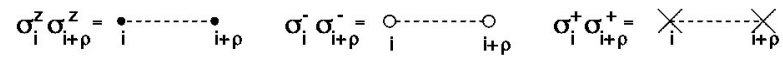

$$
\begin{aligned}
& S \longrightarrow S_{1}+S_{2}=k \sum_{p} \sigma_{p}^{+}+\sum_{p, r} b_{r} \sigma_{p}^{+} \sigma_{p+r}^{+}=\sum_{p} \underset{p}{X}+\sum_{p, r} \underset{p}{X} \underset{p+r}{X} \\
& S^{\prime \prime} \longrightarrow S_{1}^{\prime \prime}+S_{2}^{\prime \prime}=k^{\prime \prime} \sum_{m} \sigma_{m}^{-}+\sum_{m, n} b_{n}^{\prime \prime} \sigma_{m}^{-} \sigma_{m+n}^{-}=\underset{m}{\sum} \underset{m}{\circ}+\sum_{m, n}^{\sum} \underset{m}{\sim} \sim \underset{m+n}{\sim}
\end{aligned}
$$

FIG. 3. The diagrammatic representation of the ECCM formalism. The dashed lines denote the interaction terms from the Hamiltonian. The circle and the cross denote, respectively, a creation operator and a destruction operator. The straight solid lines are known as the ket lines and the wiggly solid lines are known as the bra lines.

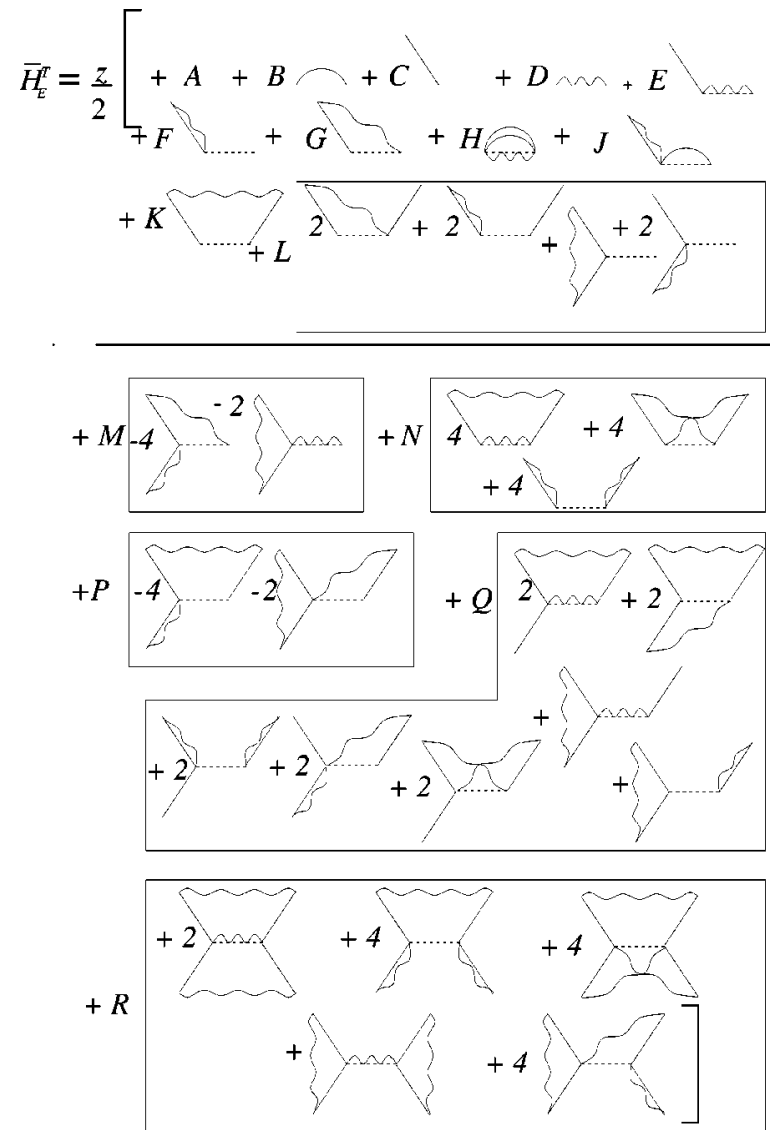

FIG. 4. The diagrams arising from the ECCM energy functional (2.4) using the Hamiltonian $H^{T}$, which yields the ground-state energy. The factors denoted by the letters are composed of functions of the angles $\alpha$ and $\beta$ and the anisotropy $\Delta$. The factor $z$ denotes the coordination number of the lattice. The diagrams that appear up to the solid line are those from the NCCM formulation.

as shown by the NCCM diagrams in Fig. 4. On the other hand, Harris ${ }^{35}$ uses a representation where the algebra for performing the CCM with respect to spin states is reformulated in terms of particle-hole states. This particular representation was employed for the purpose of identifying canceling terms and to aid the simplification and systematization of the algebra.

By contrast with these earlier diagrammatic approaches, the originality of the diagrammatic approach ${ }^{28}$ used in the present work appears at the level of the numerical calculations. Since the diagrammatic representation requires less computer memory and CPU time than the algebraic representation, higher-order truncation schemes can be implemented. There are several other advantages of using this particular approach: applying the formalism is straightforward, the terms which contribute the most in any calculation can readily be pinpointed, and the correlation function and sublattice magnetization can be expressed as sets of the diagrams which appear in the CCM energy functional arising from $H^{T}$.

Our diagrammatic technique involves casting the combinations of creation and destruction operators which appear in the CCM in a diagrammatic form, as shown in Fig. 3. The CCM SUB $n$ approximation scheme retains all configurations up to and including $n$-body correlations. Therefore, the 
SUB2 scheme retains all the one-body correlation terms, denoted by $S_{1}$ in Fig. 3, that are allowed to arise from the terms in the Hamiltonian $H^{T}$ in Eq. (3.11) which contain a singlebody creation or destruction operator, as well as all the twobody correlations, denoted by $S_{2}$ in Fig. 3 . The SUB1 scheme retains only the one-body correlations. In the present work, the correlation operators $S$ and $S^{\prime \prime}$ defined in Eqs. (2.1) and (2.3) are truncated via the SUB2 scheme as shown in Fig. 3.

As the equations that arise from the SUB2 scheme are not analytically soluble, a further restriction is placed on the maximum range of the two-body correlations from the SUB2 scheme. Therefore, the partial SUB2 approximation known as the SUB2- $n$ scheme is implemented. In the current diagrammatic formulation the SUB2- $n$ scheme retains only those two-body correlation coefficients $b_{r}$, where $r$ runs over the $n$ distinct vectors within a reference box of given size. The vectors are defined to be distinct after allowing for all lattice symmetries. For example, the linear-chain case has the property that all correlation coefficients arising from vectors of the same length are identical. However, for the squarelattice case, vectors of the same length which are not otherwise identical under the lattice symmetries yield distinct correlation coefficients. For example the vectors $(5,0)$ and $(3,4)$ have the same length but are distinct.

The one-body terms in $S_{1}$ from the SUB1 scheme simply transform a particular model state $\phi$ to an improved model state $\left|\phi^{\prime}\right\rangle$, where $|\phi\rangle$ is in the neighborhood of $\left|\phi^{\prime}\right\rangle$, via the transformation $\left|\phi^{\prime}\right\rangle=e^{S_{1}}|\phi\rangle$. The one-body terms from the SUB1 scheme are rigorously zero when the chosen model state is the same as the ground-state mean-field states, as the SUB1 scheme corresponds to a mean-field calculation. Thus, the particular cases of the $z$-aligned and $x$-aligned Néel model states for $\Delta \geqslant 1$ and $-1 \leqslant \Delta \leqslant 1$, respectively, as shown in Fig. 2, from the Hamiltonian $H^{T}$ in the local rotated axes in Eq. (3.11), yield rigorously zero one-body coefficients. More general canted model states yield very small one-body contributions. Therefore, in order to keep the number of diagrams under control, we restrict the one-body terms to give, at most, a linear contribution, which corresponds to the NCCM SUB1 scheme. Hence, the restricted SUB2 brastate parametrization can now be expressed as

$$
\begin{aligned}
\langle\widetilde{\psi}| & =\langle\phi|\left(1+S_{1}^{\prime \prime}\right) \exp \left[S_{2}^{\prime \prime}\right] \\
& =\langle\phi|\left(1+\sum_{i} k^{\prime \prime} \sigma_{i}^{-}\right) \exp \left[\sum_{i, r} b_{r}^{\prime \prime} \sigma_{i}^{-} \sigma_{i+r}^{-}\right] .
\end{aligned}
$$

Once the energy functional (2.4) arising from $\bar{H}^{T}$ is calculated, the ground-state properties can be derived by applying the stationary principle of Eq. (2.9). An example of how the diagrammatic calculation is performed can be shown by taking the expectation value of the term $\sigma_{i}^{z} \sigma_{i+\rho}^{z}$, which is a constituent of $H^{T}$. The nested commutator in Eq. (2.5) yields the following expression:

$$
e^{-S} \sigma_{i}^{z} \sigma_{i+\rho}^{z} e^{S}=\sigma_{i}^{z} \sigma_{i+\rho}^{z}+\left[\sigma_{i}^{z} \sigma_{i+\rho}^{z}, S\right]+\frac{1}{2}\left[\left[\sigma_{i}^{z} \sigma_{i+\rho}^{z}, S\right], S\right] .
$$

The correlation operator, $S_{2}$ in Fig. 3, which is described as a ket line, flips two spins in the model state $|F\rangle$ to the up position. The nested commutator in Eq. (4.3) only allows nonzero contributions, if the ket line is connected to the term $\sigma_{i}^{z} \sigma_{i+\rho}^{z}$, in the Hamiltonian. Therefore, at least one of the spins is flipped on either of the lattice sites $i$ and $i+\rho$. Consequently, a spin is flipped on one other site of the lattice.

The bra lines arising from $e^{S^{\prime \prime}}$ only yield nonzero contributions with the state described above if the final state is one of all down-pointing spins. As the bra lines flip up-pointing spins to down-pointing spins, the ends of the bra lines must be connected to ket lines or to a term in $H^{T}$ that contains creation operators. Otherwise, the destruction operators at either end of the bra line annihilate the state. The combinatorics of both the bra lines and the ket lines in devising the diagrams is taken care of by the CCM formalism. The ket lines arising from the nested commutator and the bra lines arising from $e^{S^{\prime \prime}}$ have counting factors that remove the possibility of obtaining diagrams which are equivalent under lattice symmetries.

\section{B. Diagrams arising from the ECCM formulation for macroscopic quantities}

\section{Diagrams arising from the energy functional}

The diagrammatic technique applied to the ECCM energy functional arising from the transformed Hamiltonian $H^{T}$ yields the diagrams shown in Fig. 4.

\section{Sublattice magnetization}

The sublattice magnetization or the order parameter in the Ising-Heisenberg and $X Y$-Heisenberg phases, respectively, is defined by

$$
\begin{gathered}
M^{z}=\frac{2}{N} \sum_{k \in A}\left|\left\langle U^{z} \sigma_{k}^{z} U^{z \dagger}\right\rangle\right|=-\frac{2}{N} \sum_{k \in A}\left\langle\sigma_{k}^{z}\right\rangle, \quad \Delta \geqslant 1, \\
M^{x}=\frac{2}{N} \sum_{k \in A}\left|\left\langle U^{x} \sigma_{k}^{x} U^{x \dagger}\right\rangle\right|=-\frac{2}{N} \sum_{k \in A}\left\langle\sigma_{k}^{z}\right\rangle, \quad-1 \leqslant \Delta \leqslant 1 .
\end{gathered}
$$

The inclusion of the minus sign ensures that $M^{z}$ and $M^{x}$ are positive in our rotated Néel basis, where $U^{z}$ and $U^{x}$ are the unitary operators, which rotate the local spin axes of the $z$-aligned and $x$-aligned Néel model states, respectively, to give the state $|F\rangle$. The sublattice magnetization for $\Delta \geqslant 1$ is solely that of the $z$-aligned spins $M^{z}$, and for $-1 \leqslant \Delta \leqslant 1$ the sublattice magnetization is that of the $x$-aligned spins $M^{x}$ in the present model state formulation. The formal reason for this behavior of the sublattice magnetization is given in Sec. V.

As the forms of the sublattice magnetizations $M^{z}$ and $M^{x}$ for the respective regions shown in Eq. (4.4) are equivalent, they arise from the same diagrams, which are shown in Fig. 5. Local quantities in the CCM formulation yield sizeextensive results. The diagrams for the ECCM sublattice magnetization are the same as those for the NCCM. Even though they are local quantities, the order parameters arise 


$$
\left.-\frac{2}{N} \sum_{k \in A}\left\langle\sigma_{k}{ }^{2}\right\rangle=1-2 \mathrm{kk}^{\prime \prime}-\frac{4}{\mathrm{~N}} \sum_{k \in A}\right\rangle_{k}
$$

FIG. 5. The diagrams constituting the sublattice magnetizations $M^{z}$ and $M^{x}$.

from summing correlations over the whole lattice, and in this way they are able to describe the ordering of individual spins throughout the system.

\section{Correlation function}

In order to study critical phenomena it is important to study a particular correlation function which is consistent with the definition of the order parameter. The spin-spin correlation function $g_{0}^{\alpha, \beta}$ is defined by

$$
g_{0}^{\alpha, \beta}(r) \equiv\left\langle\sigma_{k}^{\alpha} \sigma_{k+r}^{\beta}\right\rangle, \quad \alpha, \beta=x, y, z
$$

in terms of which we define the correlation function $g^{\alpha, \beta}(r)$ as follows:

$$
g^{\alpha, \beta}(r) \equiv g_{0}^{\alpha, \beta}(r)-\left\langle\sigma_{k}^{\alpha}\right\rangle\left\langle\sigma_{k+r}^{\beta}\right\rangle,
$$

where $r$ is restricted such that $k$ and $k+r$ are both on the $A$ sublattice. The translational invariance of the lattice implies, if $\alpha=\beta$ on a particular sublattice, $\left\langle\sigma_{i}^{\alpha}\right\rangle \equiv\left\langle\sigma_{i+r}^{\beta}\right\rangle$. Hence, the correlation function satisfies the fundamental property

$$
\lim _{r \rightarrow \infty} g_{0}^{\alpha, \alpha}(r)=\left(\frac{2}{N} \sum_{k \in A}\left\langle\sigma_{k}^{\alpha}\right\rangle\right)^{2} \equiv\left(M^{\alpha}\right)^{2},
$$

where $M^{\alpha}$ is the order parameter and $i$ is summed over the sublattice of $N / 2$ lattice sites. The expression for $g_{0}(r)$ in terms of the rotated canted model state is given by

$$
g_{0}(r) \equiv\left\langle U \sigma_{k}^{x} \sigma_{k+r}^{x} U^{\dagger}\right\rangle+\left\langle U \sigma_{k}^{y} \sigma_{k+r}^{y} U^{\dagger}\right\rangle+\left\langle U \sigma_{k}^{z} \sigma_{k+r}^{z} U^{\dagger}\right\rangle,
$$

where $U$ is the unitary operator in Eq. (3.6). Clearly the form of the expression in Eq. (4.8) is similar to the form of the corresponding energy functional. The same diagrams arise for $g_{0}(r)$ as for the energy functional, which is shown in Fig. 4. However, the links can now be of arbitrary length $r$. The property in Eq. (4.7) implies that in the limit $r \rightarrow \infty$, surviving diagrams from $g_{0}(r)$ can be interpreted as being topologically disconnected. Conversely, Eq. (4.6) implies that the correlation function $g(r)$ consists of the topologically connected diagrams arising from $g_{0}(r)$. Examining the topology of diagrams arising from the functional provides a straightforward way to deduce the terms that yield the sublattice magnetization and the correlation function in an ECCM calculation.

The relevant correlation functions for the regions which encompass the Ising-Heisenberg and $X Y$-Heisenberg phases, respectively, are given by

$$
\begin{aligned}
g^{z z}(r)= & \left\langle U^{z} \boldsymbol{\sigma}_{k}^{z} \boldsymbol{\sigma}_{k+r}^{z} U^{z \dagger}\right\rangle \\
& -\left\langle U^{z} \boldsymbol{\sigma}_{k}^{z} U^{z \dagger}\right\rangle\left\langle U^{z} \boldsymbol{\sigma}_{k+r}^{z} U^{z \dagger}\right\rangle, \quad \Delta \geqslant 1,
\end{aligned}
$$

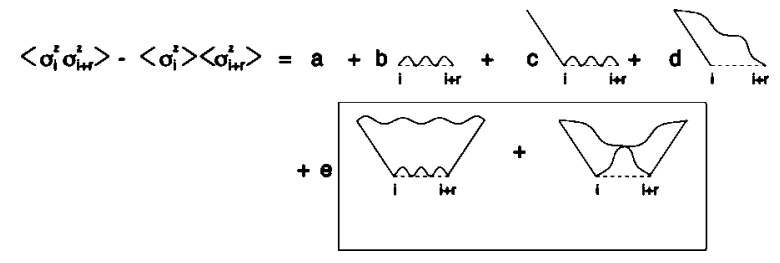

FIG. 6. The diagrams for the correlation functions, $g^{z z}$ and $g^{x x}$.

$$
\begin{aligned}
g^{x x}(r)= & \left\langle U^{x} \sigma_{k}^{x} \sigma_{k+r}^{x} U^{x \dagger}\right\rangle \\
& -\left\langle U^{x} \sigma_{k}^{x} U^{x \dagger}\right\rangle\left\langle U^{x} \sigma_{k+r}^{x} U^{x \dagger}\right\rangle, \quad-1 \leqslant \Delta \leqslant 1 .
\end{aligned}
$$

In the limit $r \rightarrow \infty$, the correlations between spins die off as shown in Eq. (4.7). The spin-spin correlation function for the region $-1 \leqslant \Delta \leqslant \infty$ has the form $\pm\left\langle\sigma_{i}^{z} \sigma_{i+r}^{z}\right\rangle$ as $\left\langle U^{z} \sigma_{i}^{z} \sigma_{i+r}^{z} U^{z \dagger}\right\rangle=\left\langle U^{x} \sigma_{i}^{x} \sigma_{i+r}^{x} U^{x \dagger}\right\rangle= \pm\left\langle\sigma_{i}^{z} \sigma_{i+r}^{z}\right\rangle$, where the sign is positive if both lattice sites are on the same sublattice and negative otherwise. Therefore, the correlation function consists of diagrams similar to the connected diagrams arising from the term $\left\langle\sigma_{i}^{z} \sigma_{i+\rho}^{z}\right\rangle$ in the energy functional $\bar{H}^{T}$. The diagrams arising from the correlation function in Eq. (4.9) are shown in Fig. 6.

The connected diagrams which appear in the NCCM correlation function are equivalent to those in Fig. 6 with the exception of those indicated in the box. However, there are also disconnected diagrams in the NCCM formulation of the correlation function. This occurs because one of the diagrams that arises from the square of the sublattice magnetization in Fig. 6 is disconnected and corresponds to a higherorder term that cannot appear in the spin-spin correlation function, due to the NCCM bra-state parametrization. Consequently, the cancellation of the disconnected diagram cannot occur when the correlation function is calculated in Eq. (4.9). However, the ECCM bra-state parametrization yields higher-order terms than the NCCM, which allows this cancellation to take place. Hence, the correlation function is solely composed of connected diagrams, which possesses the fundamental property of Eq. (4.7). In this way the importance of the cluster property, which is intrinsic in the ECCM formalism in Eq. (2.10), can be seen for the correlation function.

\section{SYMMETRIES OF THE MEAN-FIELD MODEL STATE}

The criteria for a good choice of model state in terms of the maximum overlap between the model state and the ket state have been derived by Kümmel. ${ }^{36}$ One of these criteria specifies that there should be no contribution from the onebody terms (or the SUB1 scheme) in the CCM calculation. The model states arising from the ground-state mean-field calculation satisfy this criteria. From the canted model state, the mean-field calculation yields the ground state for the $z$-aligned Néel model state in the region $\Delta \geqslant 1$ and the $x$-aligned Néel model state in the region $-1 \leqslant \Delta \leqslant 1$, as shown in Fig. 2. However, it can be shown, by applying a theorem first enunciated by $\mathrm{Xian}^{37}$ to these mean-field model states, that the ket state $|\psi\rangle$ cannot exhibit symmetry breaking at $\Delta=1$.

Xian's theorem ${ }^{37}$ states that the CCM equations provide 
at least one solution which guarantees the symmetry of the model state, if this symmetry is one of those belonging to the Hamiltonian. The theorem is proved by employing a symmetry operator $\Lambda$, which has the properties that the Hamiltonian in Eq. (3.1) commutes with $\Lambda$ and that the model state is an eigenstate of $\Lambda$.

In the case of the $z$-aligned Néel model state, the symmetry operator $\Lambda^{z}$ is given by the rotation operator

$$
\Lambda^{z}=\bigotimes_{i=1}^{N}\left[\cos \left(\frac{\chi}{2}\right) 1+i \sin \left(\frac{\chi}{2}\right) \sigma_{i}^{z}\right],
$$

where $i$ runs over all lattice sites. The order parameter for an arbitrary direction in the $X Y$ plane is defined by $M^{x y}$ $\equiv M^{x} \cos \alpha+M^{y} \sin \alpha$. The action of the rotation operator $\Lambda^{z}$ on the operators whose expectation values on the sublattice yield $M^{x y}$ is given by

$$
\Lambda^{z \dagger}\left(\sigma^{x} \cos \alpha+\sigma^{y} \sin \alpha\right) \Lambda^{z}=\sigma^{x} \cos (\alpha+\chi)+\sigma^{y} \sin (\alpha+\chi) .
$$

The only solution that guarantees the required invariance of $M^{x y}$ under rotation is $M^{x} \equiv 0$ and $M^{y} \equiv 0$, such that the $\mathrm{U}(1)_{x y}$ symmetry of the $z$-aligned Néel model state is preserved. Consequently, the theorem ${ }^{37}$ shows that $|\psi\rangle$ cannot exhibit broken $\mathrm{U}(1)_{x y}$ symmetry.

Similarly, the discrete symmetry operator for the particular case of the $x$-aligned Néel model state is given by

$$
\Lambda^{x}=\bigotimes_{i=1}^{N} \sigma_{i}^{x} .
$$

The transformation of the order parameter $M^{z}$ to $-M^{z}$ with $\Lambda^{x}$ implies that $M^{z} \equiv 0$. Thus, the $Z(2)$ symmetry of the $x$-aligned Néel model state is preserved and consequently $|\psi\rangle$ cannot exhibit broken Z(2) symmetry. Hence, it follows that the $z$-aligned Néel model state only describes the region $\Delta \geqslant 1$, where the ground state does not have broken $\mathrm{U}(1)_{x y}$ symmetry. Similarly, the $x$-aligned Néel model state only describes the region $-1 \leqslant \Delta \leqslant 1$, where the ground state does not have broken $\mathrm{Z}(2)$ symmetry.

\section{NUMERICAL RESULTS}

The diagrams in the ECCM energy functional corresponding to the Hamiltonian $H^{T}$, which arise from the SUB2- $n$ approximation scheme in Fig. 4, are used to obtain the numerical results for both the linear-chain and square-lattice cases. The qualitative behavior of the numerical results differs for the two cases. This difference can only arise from the different multiplicities of the diagrams, which themselves are due to the symmetries of the lines in the diagrams differing in these cases.

From the implementation of the NCCM SUB1 scheme it can be seen that the coefficients $k$ and $k^{\prime \prime}$ provide a negligible contribution to the overall calculation, where they are not rigorously zero for the $x$-aligned and $z$-aligned Néel model states. Therefore, with the benefit of hindsight, implementing the ECCM SUB1, which only introduces additional higherorder terms, would not be much different to the NCCM SUB1.

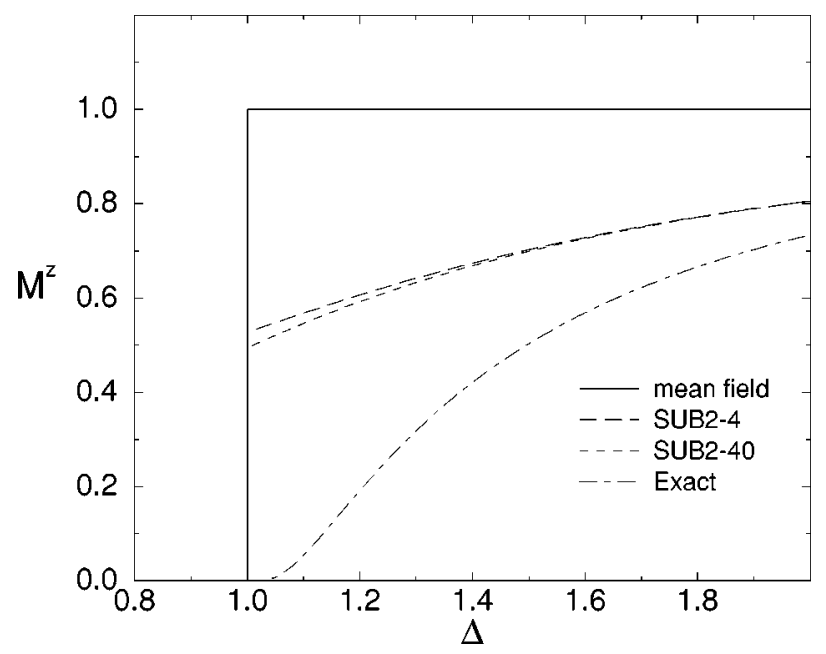

FIG. 7. The sublattice magnetization in the $z$ direction, $M^{z}$, as a function of the anisotropy $\Delta$ for the linear-chain case, from the ECCM SUB2- $n$ schemes using the $z$-aligned Néel model state, a mean-field calculation, and the exact results.

\section{A. Spin-half $X X Z$ model on the linear chain}

There are both exact results for the case of the linear chain and more appropriate numerical techniques than the CCM, such as the density matrix renormalization group and spin-wave theory, for examining more general onedimensional spin chains. However, the presence of exact results makes the linear chain an important case to gain insight into the behavior of the numerical results from the approximated ECCM formalism.

The mean-field calculation shows that both the $z$-aligned and $x$-aligned Néel model states introduce complete $z$-aligned and $x$-aligned Néel ordering respectively, at the isotropic point. Figures 7 and 8 show that the ECCM SUB2$n$ scheme fails to pick up all the quantum fluctuations in the

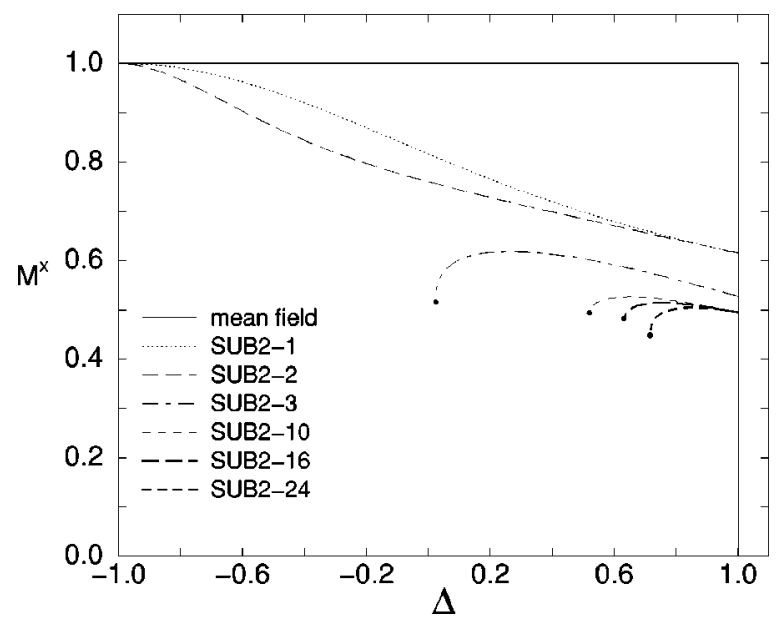

FIG. 8. The sublattice magnetization in the $x$ direction, $M^{x}$, as a function of the anisotropy $\Delta$ for the linear-chain case, from the ECCM SUB2- $n$ schemes and a mean-field calculation with the $x$-aligned Néel model state. For truncation schemes higher than the SUB2-2, solutions turn back, and so only the physical solution is shown with a solid circle indicating the turning back point, also known as the terminating point. It is known that there is no LRO over the whole region, $-1<\Delta<1$. 


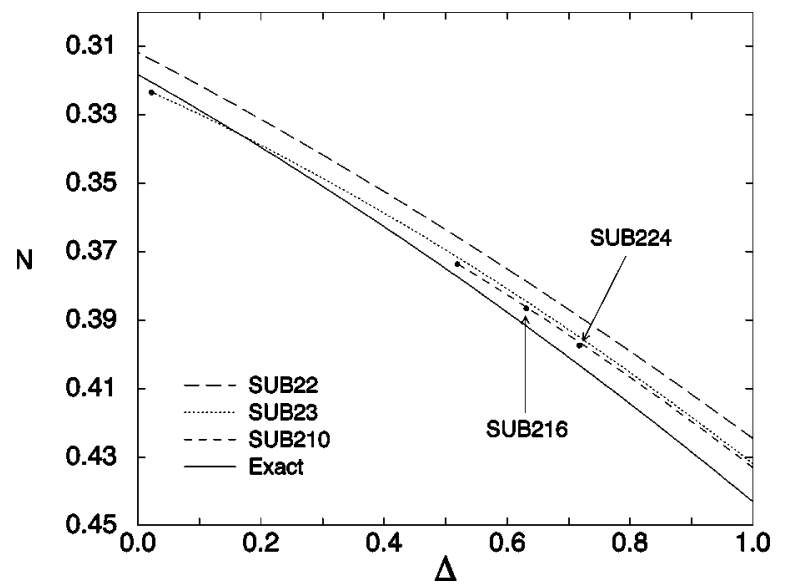

FIG. 9. The ground-state energy per spin, $E_{g} / N$, as a function of the anisotropy $\Delta$ for the linear-chain case from high-order ECCM SUB2- $n$ schemes using the $x$-aligned Néel model state, compared with the exact results.

physical system that result in the LRO being completely destroyed at the isotropic point. However, the exact order parameter from the linear-chain case exhibits subtle behavior, which, in principle, would require the implementation of extremely high-order ECCM approximation schemes.

The ECCM numerical solutions, for the quantities $M^{x}$ and $E_{g}$, from truncation schemes of orders higher than SUB2-2 with the $x$-aligned Néel model state, at the initial point $\Delta=$ -1 , do not exist for $\Delta>-1$, as shown in Figs. 8 and 9. This occurs for any scheme higher than SUB2-2 because there is a degeneracy in the numerical solutions at $\Delta=-1$, which makes it a poor choice of starting point. An alternative choice of initial point for the CCM calculation is the isotropic point, since the rotated Hamiltonians obtained from both the $x$-aligned and $z$-aligned Néel model states are equivalent at this point. These solutions turn back and become unphysical. The points at which the solutions become unphysical are known as the terminating points, and are shown in Figs. 8 and 9.

The terminating points from the SUB2- $n$ scheme based on the $x$-aligned Néel model state, at the orders that have so far been obtained, converge weakly to $1.025 \pm 0.005$ as $n$ $\rightarrow \infty$, which can be interpreted as the SUB2 terminating point. This result was obtained from the best possible mean square fit of $1 / n^{0.563}$. It indicates that the $x$-aligned Néel model state from the SUB2 approximation scheme would not yield a solution in the $X Y$-like region, where it is considered to be a better model state than the $z$-aligned Néel modelstate. This is surprising since higher orders include more of the long-range correlations in the system, and consequently should reveal more of the physical behavior of the system. The exact ground state of the linear chain in the $X Y$-like region possesses $\mathrm{U}(1)_{x y}$ symmetry, which indicates that the $x$-aligned Néel model state, as the sole input in the technique, breaks this symmetry causing solutions to terminate in the region $\Delta<1$. However, the numerical results from the ECCM for the ground-state energy and sublattice magnetization $M^{x}$ are good at low orders.

Figure 10 shows that the low-order ECCM SUB2- $n$ solutions have the same qualitative behavior as the exact groundstate energy solution, where a maximum deviation in the

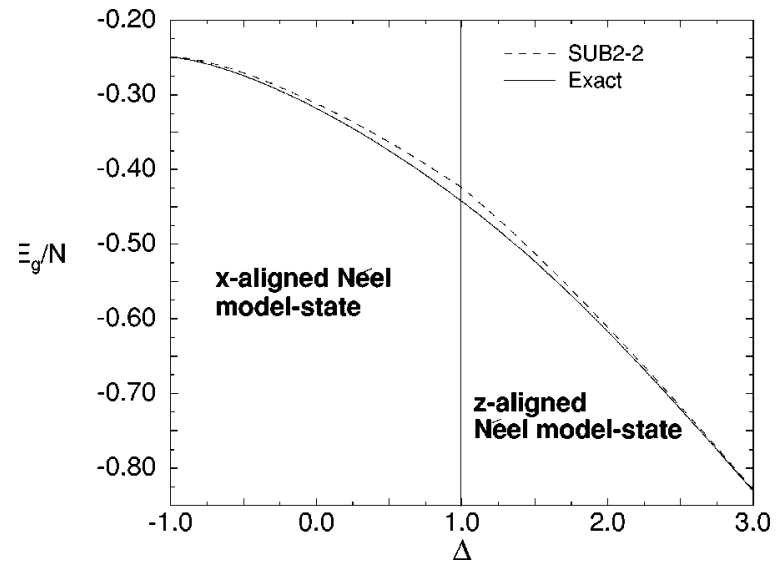

FIG. 10. The ground-state energy per spin, $E_{g} / N$, as a function of the anisotropy $\Delta$ for the linear-chain case, from the low-order ECCM SUB2-2 scheme using the $z$-aligned and $x$-aligned Néel model states in the regions $\Delta \geqslant 1$ and $-1 \leqslant \Delta \leqslant 1$, respectively, and the exact results.

energy occurs at the isotropic point. Numerical results from the NCCM LSUB $n$ scheme ${ }^{32}$ yields a numerically accurate ground-state energy at the isotropic point from an extrapolation, as shown in Table I. The LSUB $n$ scheme retains only those configurations in the correlation operator $S$ which contain any number of spin flips with respect to the model state over a localized region of $n$ contiguous sites, and which are compatible with the restriction $S_{T}^{z} \equiv \frac{1}{2} \sum_{i=1}^{N} \sigma_{i}^{z}=0$. We note that this restriction follows from the facts that $S_{T}^{z}$ commutes with $H$ and that the ground state is expected to be in the $S_{T}^{z}=0$ sector.

\section{B. Spin-half $X X Z$ model on the square lattice}

There are no exact results for the square-lattice case, which therefore presents a significant challenge for numerical techniques, particularly for the study of the nature of the expected transition at the isotropic point. Unlike the linearchain case, the square-lattice case is believed to possess LRO at the isotropic point. Evidence for this comes from a number of techniques as shown in Table II. The quantum fluctuations in the square-lattice case should be smaller than those in the linear-chain case, due to the higher coordination number of the lattice. For this reason one expects that the CCM should be better at deducing the physical behavior of the squarelattice case.

The numerical solutions for $M^{z}$ and $E_{g}$, from truncation schemes at orders higher than SUB2-12 with the $z$-aligned Néel model state terminate before the isotropic point, as shown in Figs. 11 and 12, respectively. The SUB2 terminating point of the CCM calculations based on the $z$-aligned Néel model state can be accurately determined by an ex-

TABLE I. The ground-state energy per spin for the onedimensional chain at the isotropic point, $\Delta=1$, under various CCM schemes, compared with the exact results.

\begin{tabular}{lccc}
\hline \hline NCCM SUB2 & ECCM SUB2 & NCCM LSUB $\infty$ & Exact \\
\hline-0.419 & -0.433 & -0.443149 & -0.443147 \\
\hline \hline
\end{tabular}


TABLE II. The sublattice magnetization $M^{z}$ for the two-dimensional square lattice at the isotropic point $\Delta=1$ as a fraction of the saturation value, from various CCM approximation schemes, a series expansion, a Monte Carlo simulation, and spin-wave theory (SWT).

\begin{tabular}{lccccc}
\hline \hline ECCM SUB2-12 & NCCM LSUB8 $^{a}$ & NCCM LSUB $\infty^{a}$ & Series expansion $^{b}$ & Monte Carlo & SWT $^{d}$ \\
\hline 0.689 & 0.705 & 0.622 & $0.614 \pm 0.002$ & $0.6140 \pm 0.0006$ & 0.606 \\
\hline \hline
\end{tabular}

${ }^{\mathrm{a}}$ Reference 32 .

${ }^{\mathrm{b}}$ Reference 20 .

${ }^{\mathrm{c}}$ Reference 18 .

${ }^{\mathrm{d}}$ Reference 15 .

trapolation, where the best mean-square fit of $1 / n^{2}$ gives a SUB2 terminating point at $\Delta \approx 1.03903 \pm 0.00077$. Since the $z$-aligned Néel model state only breaks the discrete Z(2) symmetry of the ground state, the SUB2- $n$ terminating points exhibit this good convergence.

Similarly, solutions starting at $\Delta=-1$ from the SUB2-20 scheme with the $x$-aligned Néel model state for $E_{g}$ and $M^{x}$ terminate in the $X Y$-like region, as shown in Figs. 12 and 13, respectively. The SUB2-20 approximation scheme is the highest we have so far investigated, as well as being the only scheme to yield a terminating solution in the $X Y$-like region. By contrast, at lower orders terminating points occur for $\Delta$ $>1$. Therefore, although the SUB2 terminating point cannot be accurately determined, it is expected to be closer to $\Delta=$ -1 than the SUB2-20 terminating point at 0.78. Consequently, it is not possible to obtain a solution from highorder SUB2- $n$ schemes that can describe the neighborhood of the isotropic point on the $\Delta<1$ side.

Unlike in the linear-chain case the existence of a solution from high-order schemes with the $x$-aligned Néel model state in the square-lattice case indicates that the continuous $\mathrm{U}(1)_{x y}$ symmetry of the ground state is broken. However, the bad convergence of the terminating points from the $x$-aligned Néel model state in the square-lattice case may indicate that the ground state is almost symmetric and, hence, a model state with $\mathrm{U}(1)_{x y}$ symmetry would be a more appropriate starting point for a CCM calculation.

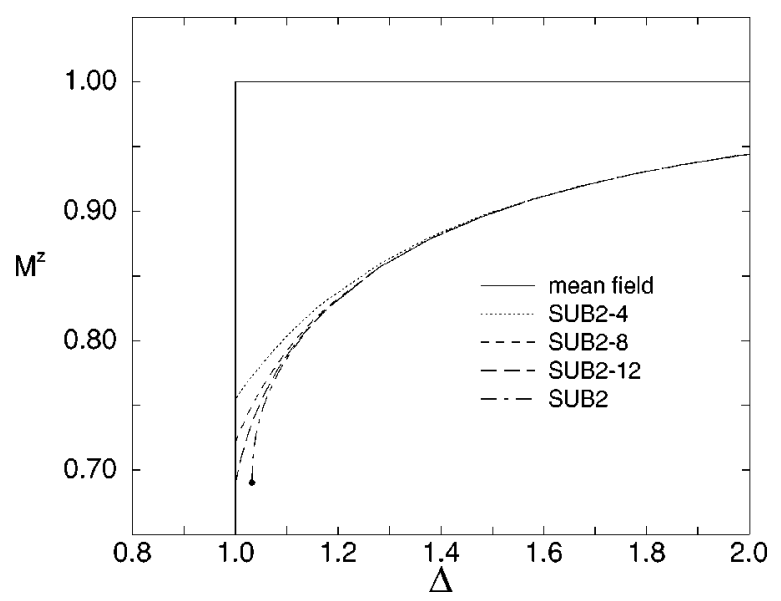

FIG. 11. The sublattice magnetization in the $z$ direction, $M^{z}$, as a function of the anisotropy $\Delta$ for the square-lattice case, from the high-order ECCM SUB2- $n$ schemes using the $z$-aligned Néel model state. The results converge at high orders of the SUB2- $n$ truncation scheme, thus giving the full SUB2 result, which corresponds to $n$ $\rightarrow \infty$.
The SUB2-12 scheme is the highest-order SUB2- $n$ scheme that yields the sublattice magnetization $M^{z}$ at the isotropic point, because at higher orders the solutions terminate before the isotropic point. The SUB2-12 scheme yields a value of $M^{z} \approx 0.689$ of the saturation value. This is an improvement upon the NCCM SUB2 value of $M^{z} \approx 0.81$, and the best NCCM result from the LSUB8 scheme, ${ }^{32}$ which yields a value of $M^{z} \approx 0.705$, when compared to other numerical techniques, as shown in Table II. The extrapolated numerical value of the magnetization from $\operatorname{LSUB} \infty, M^{z}$ $\approx 0.622$, is the result of a quadratic fit in $1 / n$ with a restricted set of three data points. The best linear fit in $1 / n$ with the same data points yields $M^{z} \approx 0.646 \pm 0.002$.

Although, as can been seen from Fig. 11, the LRO at the isotropic point decreases with increasing order, the four solutions that do not terminate before the isotropic point do not by themselves allow for a trustworthy extrapolation. Such an extrapolation should be independent of a particular scheme, as there is no known theoretical reason on how the results should converge. However, note that the nonextrapolated value of $M^{z} \approx 0.689$ of the saturation value is the best direct result available even including the best Monte Carlo calculations on a $16 \times 16$ lattice, ${ }^{38}$ a clear indication that, already at low orders, the ECCM describes the system well.

Quantitatively, the CCM yields accurate values for the ground-state energy $E_{g}$ compared to other numerical tech-

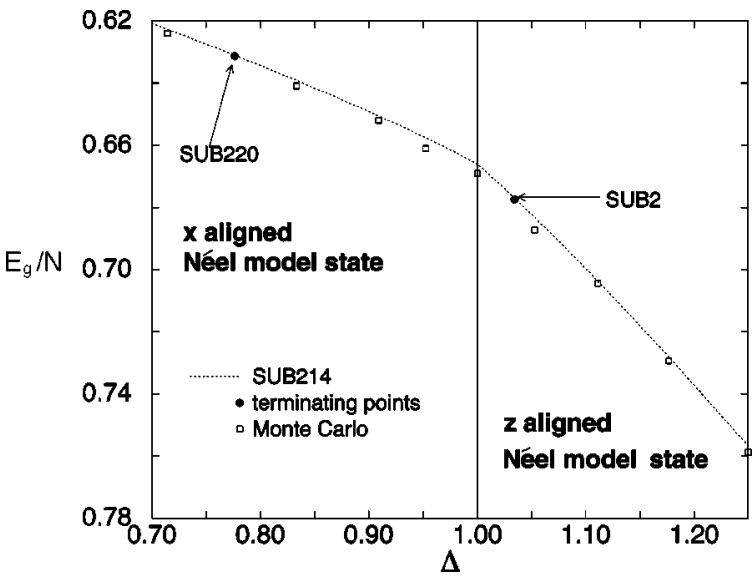

FIG. 12. The ground-state energy per spin, $E_{g} / N$, as a function of the anisotropy $\Delta$, from the $z$-aligned and $x$-aligned Néel model states in the regions $\Delta \geqslant 1$ and $-1 \leqslant \Delta \leqslant 1$, respectively, for the square-lattice case. The terminating points of the solutions from the ECCM full SUB2 and SUB2-20 approximation schemes, in the regions $\Delta \geqslant 1$ and $-1 \leqslant \Delta \leqslant 1$, respectively, are indicated by solid circles. 


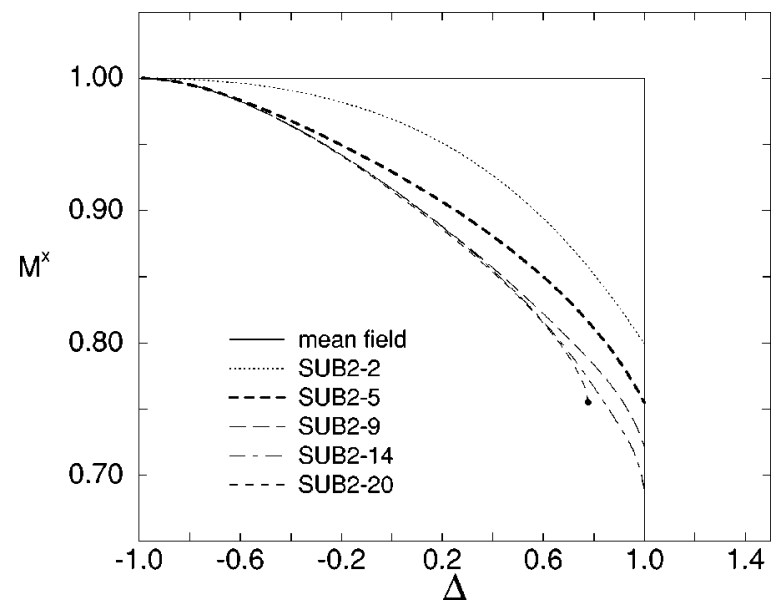

FIG. 13. The sublattice magnetization in the $x$ direction, $M^{x}$, as a function of the anisotropy $\Delta$ for the square-lattice case, from a mean-field calculation and high-order ECCM SUB2- $n$ schemes using the $x$-aligned Néel model state.

niques as shown in Table III. The NCCM SUB2 scheme ${ }^{27}$ at the isotropic point yields a ground-state energy of $E_{g} \approx$ -0.651 , which the ECCM SUB2 scheme improves upon, via an extrapolation with values that converge well, with a numerical result of $E_{g} \approx-0.667$. The calculation of the ground-state energy from the NCCM LSUB $n$ schemes $^{32}$ is extremely accurate when compared to the best Monte Carlo result $^{18}$ where the limit $n \rightarrow \infty$ is equivalent to including the complete set of many-body configurations.

\section{Canted model state in the correlated ECCM SUB2-n calculation}

The effect of the canted model state, which has two degrees of freedom, in a CCM calculation is examined here. In practice, the spins in the canted model state are restricted to point in one direction on a particular sublattice. Nevertheless, for the spin-half $X X Z$ model, the CCM with the canted model state provides a good analysis of the effect of the model state in these calculations. In terms of the numerical calculations, performing the CCM with the canted model state is a new approach, since the earlier CCM calculations for spin systems have chosen predetermined model states with no free parameters, and with the choice based on either prior knowledge or classical behavior. The use of the canted model state allows the extra freedom to partially tailor the model state to the specific value of the anisotropy parameter. This is achieved by the application of the stationary equation (2.9) and the variation with respect to the angles $\alpha$ and $\beta$, which define the model state, where the minimum energy is

TABLE III. The ground-state energy per spin for the twodimensional square lattice at the isotropic point $\Delta=1$ from various CCM schemes, compared with the results of a Monte Carlo simulation.

\begin{tabular}{lcc}
\hline \hline ECCM SUB2 & NCCM LSUB ${ }^{\mathrm{a}}$ & Monte Carlo $^{\mathrm{b}}$ \\
\hline$-0.6676 \pm 0.0005$ & $-0.66968 \pm 0.00004$ & $-0.669437 \pm 0.000005$
\end{tabular}

${ }^{\mathrm{a}}$ Reference 32 .

${ }^{\mathrm{b}}$ Reference 18 .

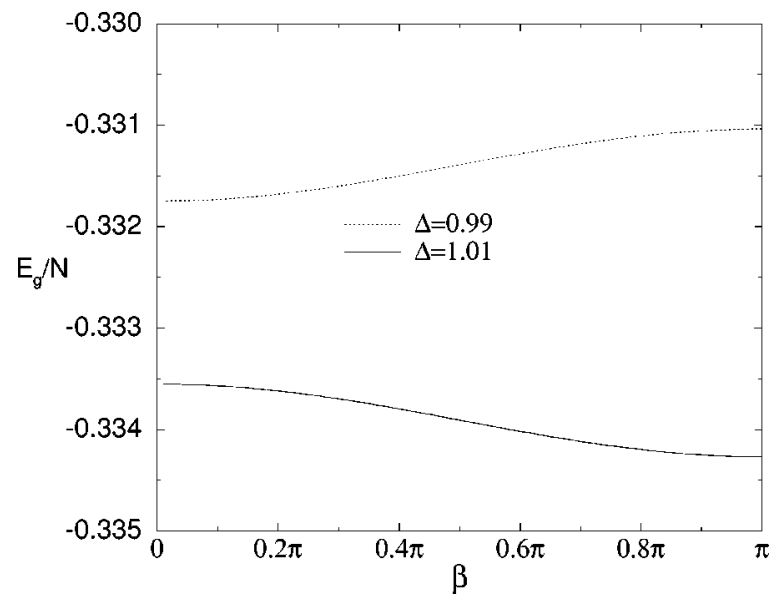

FIG. 14. The ground-state energy per spin, $E_{g} / N$, as a function of the orientation of nearest-neighbor spins, $\beta$, in a model state, which is restricted to the canted model state space, for the linearchain case, when the relative angle $\alpha$ is $\pi$ (Néel state). The examination of the model-state space is performed in both the $X Y$-like and Ising-like regions in the neighborhood of the isotropic point, using the converged ECCM full SUB2 values.

automatically chosen for $\alpha$ and $\beta$ at each value of the anisotropy $\Delta$. Therefore, we employ this particular form for the numerical CCM calculation, in the hope that it will choose model states which lie closest to the true ground state.

\section{Behavior of the canted model state with varying angles}

For the calculations with varying anisotropy, the angles $\alpha$ and $\beta$ are free to vary. The model states that arise from the ECCM SUB2- $n$ calculation in this case are the same as those from the mean-field calculation, as shown in Fig. 2, for $-1 \leqslant \Delta \leqslant \infty$. This is also illustrated by Fig. 14 in the neighborhood of the isotropic point. As discussed in Sec. V, these mean-field model states yield the CCM ground state that preserves either the $\mathrm{U}(1)_{x y}$ or $\mathrm{Z}(2)$ symmetry of the Hamiltonian.

Kümmel's work ${ }^{36}$ provides a guideline to examine the effect of the mean-field model states in the CCM calculation, if translational invariance of the rotated model state $|F\rangle$ is assumed. It can be shown that the overlap condition for the mean-field model states is maximized if the sum, over the whole lattice, of the two-body correlations, which is defined in Fig. 3 as $S_{2}$, obeys $\Sigma_{r} b_{r}<1 / 2$. This result is extended to show that $\Sigma_{r} b_{r} \rightarrow 1 / 2$ indicates a phase transition point. As $\Delta \rightarrow 1$ this condition holds for the z-aligned Néel model state in the linear-chain case and only approximately holds for the square-lattice case. For the square-lattice case, this may indicate the numerical instability of the solution as $\Delta \rightarrow 1$ and consequently the need for a model state with greater overlap in this region.

The solution for the energy at the isotropic point has a continuous degeneracy in terms of the angle $\beta$, for a fixed value of $\alpha=\pi$ which yields the set of all model states with Néel symmetry. However, away from the isotropic point there is a unique minimum in the solution. Therefore, in practice, by choosing a fixed angle $\beta$, we break the $\mathrm{SU}(2)$ symmetry at the isotropic point. In doing so, LRO is intro- 
duced in the direction specified by the angle $\beta$, which is diminished by the correlations from a CCM calculation.

\section{Behavior of the canted model state with fixed angles}

The effect of particular model states that require the inclusion of one-body terms in the CCM calculation is examined here. In the Ising-like region, for the angle $\beta$ set to $\pi$, the angle $\alpha$ is fixed to various values which define model states in the neighborhood of the $z$-aligned Néel state, i.e., $\alpha \sim \pi$. As $\alpha$ is decreased, each numerical solution for the energy in the square-lattice case, from the highest-order ECCM SUB2- $n$ scheme, is successively higher and terminates earlier than the previous solution. Furthermore, for model states defined by $\alpha \leqslant \pi / 2$, a solution cannot be recovered.

These non-mean-field model states, in the neighborhood of the $z$-aligned Néel model state, require one-body terms in the CCM calculation. Hence, the ground state can exhibit broken $\mathrm{U}(1)_{x y}$ symmetry. ${ }^{37}$ However, these model states are bad choices in comparison to the symmetry-preserving mean-field model states, due to the poor qualitative and quantitative behavior of the numerical solutions for the energy. Moreover, due to the termination of these numerical solutions well before $\Delta=1$, broken $\mathrm{U}(1)_{x y}$ symmetry does not physically occur in this region. Thus, the symmetrybreaking nature of these poor choices of model state cannot be utilized. It seems that non-mean-field model states with one-body correlations try to approach the mean-field model states and, in doing so, do a worse job at describing the system.

\section{Nontrivial behavior of the canted model state in the Ising limit}

We now examine the effect of the canted model states in the Ising limit. As shown in Fig. 14, for the Ising-like region of the linear-chain case the lowest energy, and therefore the optimum model state to yield the ground-state energy, is the $z$-aligned Néel state. Initially, in the Ising-like region the periodicity of the solution for the ground-state energy in terms of the angle $\beta$ is $2 \pi$, when $\alpha$ is fixed to $\pi$, as shown in Fig. 15 for the linear-chain case and with minima at $\pi \bmod (2 \pi)$. In the Ising limit the solution asymptotically develops a periodicity of $\pi$ in the angle $\beta$. The states at $\beta$ $=0 \bmod (2 \pi)$, which define the $x$-aligned Néel state, become degenerate with those at $\beta=\pi \bmod (2 \pi)$, which define the $z$-aligned Néel state. Interestingly, this analysis reveals that in the Ising limit, the $z$-aligned Néel ground state is obtained from the the $x$-aligned Néel model state with large one-body coefficients $k$.

\section{DISCUSSION AND CONCLUSIONS}

The diagrammatic technique has made possible the implementation of high-order SUB2- $n$ schemes in the ECCM formulation, since it requires less computer memory and CPU time than performing the CCM with algebraic methods. This is because the multiplicities of the diagrams are only determined once and can thereafter be used throughout the calculation. For the case of the square lattice, accurate numerical results in comparison to other numerical techniques have been obtained for the region $-1<\Delta<\infty$. In particular, at the

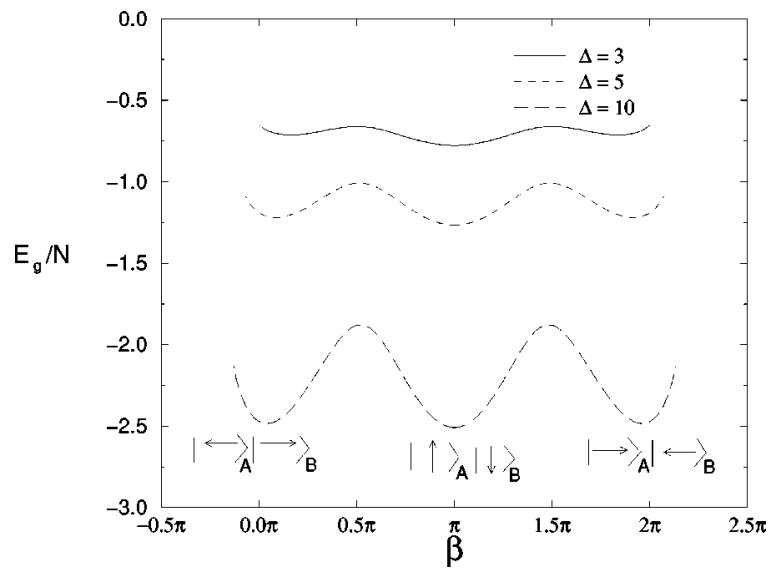

FIG. 15. The ground-state energy per spin, $E_{g} / N$, as a function of the overall orientation of nearest-neighbor spins $\beta$, when $\alpha$ is fixed at $\pi$, for the linear-chain case. The examination of the modelstate space is performed in the Ising-like region for increasing values of the anisotropy, using the converged ECCM full SUB2 values.

isotropic point the most accurate value for the order parameter of $M^{z} \approx 0.689$ for any nonextrapolated CCM calculation has been obtained. This suggests that the ECCM SUB2-n scheme describes the wave function better than previous NCCM formulations at similar levels of truncation.

The qualitative behavior of the numerical results indicate that the present CCM treatment requires further improvement for the study of phase transitions. In the case of the linear chain the ECCM fails to describe the system for $|\Delta|$ $\leqslant 1$. For the square-lattice case there is a failure to describe the region near to the isotropic point. Alternatively, the manner in which the numerical solutions become unphysical provides further knowledge of the system and the ECCM.

It is intuitively clear that a good choice of model state in a CCM calculation should be as close as possible to the exact ground state or, alternatively, share the same underlying symmetries as the exact ground state, depending on the system under consideration. The high-order SUB2- $n$ ECCM numerical results show that the important facet of a good choice of model state is dependent on the extent to which the system is ordered at the isotropic point and in the $X Y$-like region. The linear-chain case with both the $z$ - and $x$-aligned Néel model states and the square-lattice case with the $x$-aligned Néel model state indicate that when either LRO does not exist or the value of the order parameter may be quite small, it is important to chose a model state which does not artificially break the symmetry of the Hamiltonian.

Alternatively, the square-lattice case with the $z$-aligned Néel model state suggests that if the system has broken symmetry, the large quantum fluctuations that result from the massless excitations could also cause solutions to terminate. In this case, it is more important to chose a model state which is as close as possible to the exact ground state. This problem of correlations becoming too large seems to occur generally in quantum many-body techniques. Advantageously, introducing broken symmetry into such a system yields the benefit of not having to perform high-order calculations to approximate the true ground state well.

As shown above, our analysis, as it stands, requires prior knowledge of the system. Although we know from other 
numerical techniques ${ }^{15-20}$ that the square-lattice Heisenberg model possesses LRO, the value of the order parameter might be too small for the ECCM to describe the isotropic point. Therefore, the best CCM approach for this system is to define a model state with a flexible parametrization, so that it does not artificially break the symmetry of the Hamiltonian, and in so doing can give indications of possible symmetry breaking by examining the effect of correlations on the order parameter. In order to achieve this end the model-state parametrization should yield a mean-field solution in which the order parameter smoothly and continuously tends to zero at the isotropic point. In this way the ECCM formulation can be extended over the whole regime to include the long-range correlations at the critical point.

\section{ACKNOWLEDGMENTS}

We thank S. Fantoni for a useful discussion. One of us (R.F.B.) gratefully acknowledges support for this work in the form of a research grant from the Engineering and Physical Sciences Research Council (EPSRC) of Great Britain.
*Electronic address: R.F.Bishop@umist.ac.uk

${ }^{1}$ J.S. Arponen, Ann. Phys. (N.Y.) 151, 311 (1983).

2 J.S. Arponen, R.F. Bishop, and E. Pajanne, Phys. Rev. A 36, 2519 (1987); 36, 2539 (1987).

${ }^{3}$ R.F. Bishop and J.S. Arponen, Int. J. Quantum Chem.: Quantum Chem. Symp. 24, 197 (1990).

${ }^{4}$ J.S. Arponen and R.F. Bishop, Ann. Phys. (N.Y.) 207, 171 (1991).

${ }^{5}$ J.S. Arponen and R.F. Bishop, Ann. Phys. (N.Y.) 227, 275 (1993); 227, 334 (1993).

${ }^{6}$ F. Coester, Nucl. Phys. 7, 421 (1958); F. Coester and H. Kümmel, ibid. 17, 477 (1960).

${ }^{7}$ J. Čižek, J. Chem. Phys. 45, 4256 (1966); Adv. Chem. Phys. 14, 35 (1969).

${ }^{8}$ H. Kümmel, K.H. Lührmann, and J.G. Zabolitzky, Phys. Rep., Phys. Lett. 36C, 1 (1978).

${ }^{9}$ R.F. Bishop and K.H. Lührmann, Phys. Rev. B 17, 3757 (1978).

${ }^{10}$ R.F. Bishop and H. Kümmel, Phys. Today 40 (3), 52 (1987).

${ }^{11}$ R.J. Bartlett, J. Phys. Chem. 93, 1697 (1989).

${ }^{12}$ R.F. Bishop, Theor. Chim. Acta 80, 95 (1991).

${ }^{13}$ R.F. Bishop, in Microscopic Quantum Many-Body Theories and Their Applications, edited by J. Navarro and A. Polls, Lecture Notes in Physics, Vol. 510 (Springer, Berlin, 1988), p. 1.

${ }^{14}$ R.B. Laughlin, in Proceedings of the Inauguration Conference of the Asia-Pacific Center for Theoretical Physics, Seoul National University, Korea, edited by Y.M. Cho, J.B. Hong, and C.N. Yang (World Scientific, Singapore, 1998).

${ }^{15}$ P.W. Anderson, Phys. Rev. 86, 694 (1952); R. Kubo, ibid. 87, 568 (1952).

${ }^{16}$ J. Kanamori and K. Yoshida, Prog. Theor. Phys. 14, 423 (1955).

${ }^{17}$ T. Oguchi, Phys. Rev. 117, 117 (1960).
${ }^{18}$ A.W. Sandvik, Phys. Rev. B 56, 11678 (1997).

${ }^{19}$ R.R.P. Singh, Phys. Rev. B 39, 9760 (1989).

${ }^{20}$ Z. Weihong, J. Oitmaa, and C.J. Hamer, Phys. Rev. B 43, 8321 (1991).

${ }^{21}$ M.R. Li, Y.J. Wang, and C.D. Gong, Z. Phys. B 102, 129 (1997).

${ }^{22}$ H.A. Bethe, Z. Phys. 71, 205 (1931).

${ }^{23}$ L. Hulthén, Ark. Mat. Astron. Fys. 26, 11 (1938).

${ }^{24}$ R. Orbach, Phys. Rev. 112, 309 (1958); C.N. Yang and C.P. Yang, ibid. 150, 321 (1966); 150, 327 (1966).

${ }^{25}$ R.J. Baxter, J. Stat. Phys. 9, 145 (1973).

${ }^{26}$ N.D. Mermin and H. Wagner, Phys. Rev. Lett. 17, 1133 (1966).

${ }^{27}$ R.F. Bishop, J.B. Parkinson, and Y. Xian, Phys. Rev. B 43, 13782 (1991).

${ }^{28}$ N.E. Ligterink, N.R. Walet, and R.F. Bishop, Ann. Phys. (N.Y.) 267, 97 (1998).

${ }^{29}$ M. Roger and J.H. Hetherington, Phys. Rev. B 41, 200 (1990).

${ }^{30}$ R.F. Bishop, R.G. Hale, and Y. Xian, Phys. Rev. Lett. 73, 3157 (1994).

${ }^{31}$ C. Zeng, I. Staples, and R.F. Bishop, J. Phys. Condens. Matter 7, 9021 (1995).

${ }^{32}$ C. Zeng, D.J.J. Farnell, and R.F. Bishop, J. Stat. Phys. 90, 327 (1998).

${ }^{33}$ D.J.J. Farnell, S.E. Krüger, and J.B. Parkinson, J. Phys. Condens. Matter 9, 7601 (1997).

${ }^{34}$ J. Hubbard, Proc. R. Soc. London, Ser. A 240, 539 (1957).

${ }^{35}$ F.E. Harris, Phys. Rev. B 47, 7903 (1993).

${ }^{36}$ H.G. Kümmel, Nucl. Phys. A317, 199 (1979).

${ }^{37}$ Y. Xian, in Condensed Matter Theories, edited by M. Casas, M. de Llano, J. Navarro, and A. Polls (Nova Science, Commack, New York, 1995), Vol. 10, p. 541.

${ }^{38}$ M.C. Bounara and S. Sorella, Phys. Rev. B 57, 11446 (1998). 